\title{
ARTICLE
}

\section{Influence of the chromatin density on the number of direct clustered damages calculated for proton and alpha irradiations using a Monte Carlo code}

\author{
Morgane Dos Santos ${ }^{\mathrm{a}, \mathrm{c}^{*}}$, Carmen Villagrasa ${ }^{\mathrm{a}, \mathrm{c}}$, Isabelle Clairand ${ }^{\mathrm{a}, \mathrm{c}}$ and Sebastien Incerti ${ }^{\mathrm{b}, \mathrm{c}}$ \\ ${ }^{a}$ IRSN, Institut de Radioprotection et de Sureté Nucléaire, BP17, 92962 Fontenay-aux-Roses; ${ }^{b}$ Université de Bordeaux 1, \\ CNRS/IN2P3, Centre d'Etudes Nucléaires de Bordeaux Gradignan, CENBG, chemin du solarium, BP 120, 33175 Gradignan, \\ France; ${ }^{c}$ The authors are members of the Geant4-DNA collaboration
}

\begin{abstract}
The aim of this work is to evaluate the influence of the DNA density on the number of direct DNA clustered damages created by protons and alphas. Some simulations of the energy deposition were performed within two detailed DNA geometrical target models implemented in the Geant4 Monte Carlo code. An adapted DBSCAN algorithm was then used in order to determine the clustered damages.
\end{abstract}

Keywords: DNA geometry; clustering; direct DNA damages; Monte Carlo simulations

\section{Introduction}

Understand and predict the biological effects of ionising radiation remains a challenge in radiobiology research. To date, there are evidences that DNA is the privileged target giving rise to irradiation damages to cells [1]. The energy deposited by the ionising particles produces critical DNA damages such as base damages or strand breaks, nevertheless it is generally accepted that DNA double strand breaks (DSB) are the main responsible for chromosomal aberrations and cell death [2]. Nowadays, Monte Carlo track calculations are the only method to accurately simulate the energy deposition by ionizing radiation in such small biological structures. In this work, we used the Geant4 Monte Carlo toolkit, version 9.5 , to carry out the simulations [3-4].

Two detailed geometries of cell nucleus, representing a fibroblast and an endothelium, were implemented as the target of proton and alpha irradiations in order to evaluate the influence of the DNA density and the radiation qualities on the number and complexity of damages. This evaluation is based on a relative quantification of DNA clustered damages created on the implemented DNA geometries and revealed thanks to an adapted clustering algorithm.

\section{Material and method}

\subsection{The Monte Carlo code GEANT4}

Geant4 has been recently extended [5-8] to handle micro and nanodosimetry applications by the use of

*Corresponding author. Email: dos-santos@imnc.in2p3.fr physical processes called Geant4-DNA, that allow simulations with nanometric precision on the energy deposited by ionizing radiation on sub-cellular studies. Specific cross sections were introduced in the code in order to take into account all processes leading to energy deposition or non-neglected direction changes in the track of electrons, protons and alpha particles (and their different charge states) interacting with liquid water. Indeed, liquid water is commonly used to represent biological material, because it is the most abundant molecule present in biological cells. Thereby, different models can be chosen to simulate inelastic collisions (ionisations and excitations), charge transfer and elastic scattering (the later for electrons). The Monte Carlo toolkit is then suitable to transport protons between 1 $\mathrm{keV}$ and $100 \mathrm{MeV}$, alpha particles between $1 \mathrm{keV}$ and $400 \mathrm{MeV}$ and electrons between few eVs and $1 \mathrm{MeV}$.

\subsection{DNA geometrical target models}

The DNA target models implemented in Geant4 represent the whole genome of a human fibroblast and a human endothelium cell nucleus in the G0/G1 phase where the genome occupies the total cell nucleus volume in a rather homogeneous manner. Each nucleus is enveloped by an ellipsoid with half axis of 9.85, 7.1 and $2.5 \mu \mathrm{m}$ for the fibroblast and 9.55, 5.5 and $1 \mu \mathrm{m}$ for the endothelium. The nucleus volume is divided in 46 regions with a volume proportional to the chromosome's size. The main difference between these two cell nuclei is the relative volume occupied by the DNA as the genome content is the same and the total cell nucleus volume is different: the volume of the fibroblast cell nucleus is indeed three times larger than for the endothelium. The DNA molecule is then more compact 
in the endothelium cell nucleus. The relative volume occupied by DNA is then $1.43 \%$ for the endothelium nucleus and $0.42 \%$ for the fibroblast. The geometry of the total DNA molecule (about $6 \mathrm{Gbp}$ ) within the cell nucleus is divided in five compaction levels based on the PARTRAC geometrical model [9,10]: DNA double helix, nucleosomes, chromatin fiber, chromatin fiber loop and chromosome territories.

The basic element of the geometry is a nucleosome (Figure 1a) that is composed of a cylinder representing the histone proteins wrapped by roughly two turns of DNA double helix containing about 200 amino bases and the sugar phosphate corresponding region. From this element a solenoid chromatin fiber of 90 nucleosomes (Figure 1b) with a diameter of $31 \mathrm{~nm}$ is built. Four of this chromatin fibers are used to form loops, assembled in a diamond shape (Figure 1c). This "simple loop" is composed of $72 \mathrm{kbp}$ and can be used to form another level of compaction used in the case of the fibroblast description with a form close to a "flower" (Figure 1d) composed of 0.5 Mbp. The "Simple loop" is used to fill the endothelium chromosome territories and the "flower" loop is used to fill the fibroblast chromosome territories. Further details about the DNA models are given in [11].

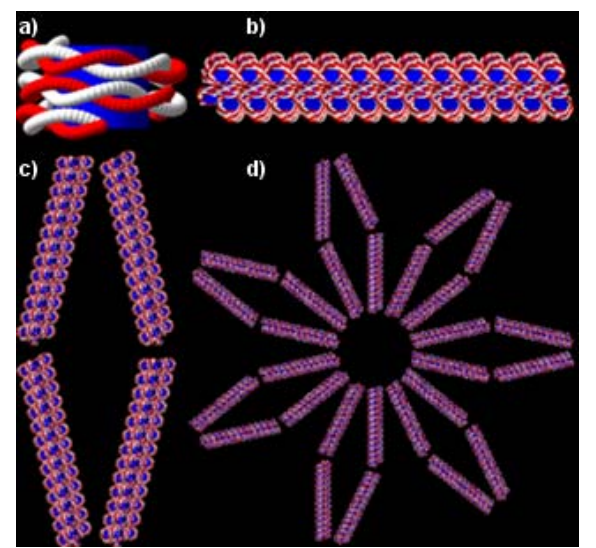

Figure 1. a: nucleosome, b: chromatin fiber, c: simple loop, d: flower.

\subsection{Simulated beam}

In the present work, simulations with protons between 0.5 and $50 \mathrm{MeV}$ (1.245 to $41.3 \mathrm{keV} / \mu \mathrm{m})$ and alphas between 5 and $50 \mathrm{MeV}$ (15.17 to $88.55 \mathrm{keV} / \mu \mathrm{m})$ were performed. The proton and alpha beam were simulated parallel to the lower axis of the nucleus $(\mathrm{z})$, the maximal thickness crossed by the beam being $5 \mu \mathrm{m}$ for the fibroblast and $2 \mu \mathrm{m}$ for the endothelium. The position $\mathrm{x}$ and $\mathrm{y}$ was chosen randomly in order to cover the entire surface of the nucleus. The number of simulated events was calculated in order to obtain statistically steady results in term of DSB candidates per particle and per micrometer. Indeed, our results are normalized by the average length crossing. The results obtained do not vary more than $2 \%$.

\subsection{Clustering algorithm}

On our previous work [12], a DBSCAN clustering algorithm allowing the calculation of the absolute number (direct + indirect) of early DNA damages (DSB and SSB) has been adapted. In that previous work, tracks were simulated in a homogeneous liquid water containment and only a fraction of the energy transfer points (16\% randomly chosen) was selected to be used on the DBSCAN algorithm for calculating the DNA breaks.

In this present work, the implementation of a detailed DNA geometry, helps to make in a more realistic manner the selection of the energy transfer points suitable for producing strand breaks.

These energy transfer points are those located on the backbone region (sugar / phosphate group). All ionizations and excitations of the media located on the backbone region were thus taken into account by the clustering algorithm. The relative position of all these energy transfer points was analysed with an adapted DBSCAN clustering algorithm [12] in order to reveal clusters of energy deposition that could correspond to a simple strand break (SSB) or a DSB. This method gives also information about the complexity of the obtained clusters.

The parameters used by the clustering algorithm take into account the fact that the separation of a maximum of $3.2 \mathrm{~nm}$ (that corresponds to roughly the distance between 10bp) can be at the origin of a DSB if the points are located in opposite strands [11].

From these considerations, three types of resulting clusters can be distinguished and associated to a classification of possible damages that has been reported in Table 1.

Table 1. Different types of DNA clustered damages.

\begin{tabular}{|c|c|c|}
\hline $\begin{array}{l}\text { Type of } \\
\text { cluster }\end{array}$ & Definition & Name \\
\hline $\begin{array}{c}\text { Isolated } \\
\text { interaction } \\
\text { points } \\
\text { Simple } \\
\text { cluster }\end{array}$ & $\begin{array}{l}\text { Interaction points located } \\
\text { more than } 10 \mathrm{bp} \text { away from } \\
\text { another points } \\
\text { Interaction points located on } \\
\text { the same strand and } \\
\text { separated by less than } 10 \mathrm{bp}\end{array}$ & $\begin{array}{c}\text { SSB } \\
\text { candidates } \\
\left(\mathrm{SSB}_{\text {cand }}\right)\end{array}$ \\
\hline $\begin{array}{l}\text { Complex } \\
\text { cluster }\end{array}$ & $\begin{array}{l}\text { Interaction points for which } \\
\text { at least one of them is } \\
\text { located on a different strand } \\
\text { and separated by less than } \\
10 \mathrm{bp}\end{array}$ & $\begin{array}{c}\text { DSB } \\
\text { candidates } \\
\left(\mathrm{DSB}_{\text {cand }}\right)\end{array}$ \\
\hline
\end{tabular}

\section{Results and discussion}

In this section only the results concerning the potential candidates to DSB have been reported as the conclusions about the SSB candidates are equivalent.

\subsection{Total DSB candidates}

Figure 2 shows the evolution of the total number of DSB candidates per particle and micrometer for each 
type of cell nucleus as a function of the LET of the projectile.

The conclusions concerning the influence of the DNA density are the same for alpha and proton irradiations (protons were studied in more details in our previous work [11]). The total number of DNA clustered damages increases with increasing radiation LET due to the increasing of the track density. Moreover, more damages have been found in the endothelium cell nucleus than in the fibroblast for a given LET. The DNA density in the cell nucleus being higher for the endothelium than for the fibroblast, the probability to obtain energy transfer points located on the backbone region is then higher for the endothelium cell.

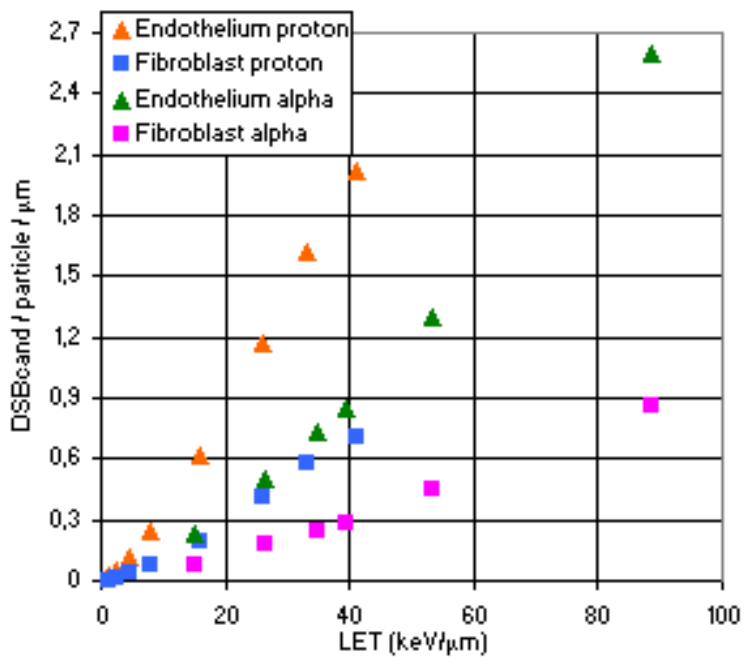

Figure 2. $\mathrm{DSB}_{\text {cand }}$ per particle and micrometer for each type of cell nucleus.

Concerning the influence of the radiation quality, for a given LET and a given cell nucleus, more possible damages were found after a proton irradiation than for an alpha irradiation.

Table 2 reports the difference in the number of resulting clustered damages created by protons versus alphas (in \%) depending on the cell nuclei.

Table 2. Difference in the number of clustered damages between proton and alpha (DSBcand_a / DSBcand_p in \%).

\begin{tabular}{cccc}
\hline $\begin{array}{c}\text { LET } \\
\text { proton } \\
(\mathrm{keV} / \mu \mathrm{m})\end{array}$ & $\begin{array}{c}\text { LET } \\
\alpha \\
(\mathrm{keV} / \mu \mathrm{m})\end{array}$ & $\begin{array}{c}\text { \% of } \\
\text { difference in } \\
\text { endothelium } \\
\text { cell }\end{array}$ & $\begin{array}{c}\text { \% of } \\
\text { difference in } \\
\text { fibroblast } \\
\text { cell }\end{array}$ \\
\hline 41.32 & 39.3 & 57.61 & 59.35 \\
33.25 & 34.89 & 55.04 & 58.45 \\
26.08 & 26.41 & 57.09 & 57.29 \\
15.89 & 15.17 & 63.43 & 60.47 \\
\hline
\end{tabular}

As it can be seen from this table, the difference in the number of DSB created by protons or alphas (between 55 and $63 \%$ ) seems to be independent of their LET (on this range of values) and the cell nucleus type. The probability of creating a DSB is always higher for proton irradiation than for alphas. Indeed, as the energy communicated to secondary electrons depends on the kinetic energy of the primary particle, for a same LET, more energetic electrons are created after alpha irradiations. These electrons have lower interaction cross sections and consequently a higher mean free path leading to a more sparse energy transfer point distribution. Thus, the probability to have energy transfer points located on the backbone region close enough to give rise to DSB candidates is lower after an alpha irradiation [13].

\subsection{Complexity of DSB candidates}

Concerning the complexity of the potential DSB candidates revealed by the clustering algorithm, in Figure 3, we plotted the number of $\mathrm{DSB}_{\text {cand }}$ per alpha particle and micrometer as a function of their size for three different LET of the alpha beam.

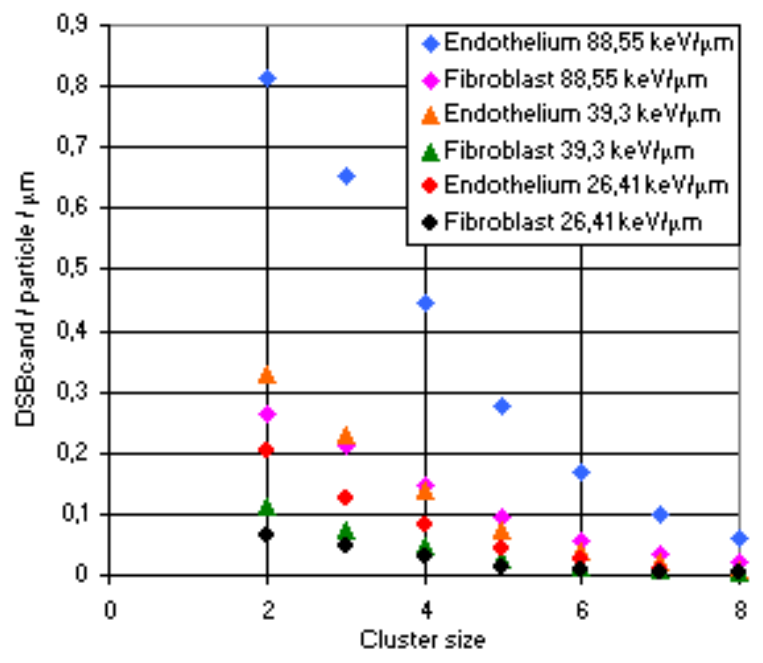

Figure 3. Complexity of DNA clustered damages induced by alphas for three different LET.

As in the case of proton irradiations [11], the frequency of the clusters obtained for a given cluster size increases with increasing radiation LET due to a higher track density.

Comparing the cell nuclei, more damages were systematically found for the endothelium cell nuclei for clusters containing between 2 and 8 energy transfer points. Thus, the increase of the DNA density seems to increase the quantity and the complexity of direct clustered damages and can be a factor contributing to the difficulty of the DNA repair. Nevertheless, it is important to remind that our geometrical cell nucleus models are simplified models. The DNA molecule is distributed homogeneously on the two models whereas, in reality, the DNA can be more or less compact in certain regions of the nucleus (euchromatin and heterochromatin regions). A study concerning the influence of the euchromatin/heterochromatin regions is in progress. Thus, in this present work, the purpose is to study the influence of the DNA density, not directly correlate this result to the influence of the cell type. 
Concerning the relative frequency between different cluster sizes for different LET, the results show the same trend for the two types of cell nuclei. Table 3 reports the frequency of the $\mathrm{DSB}_{\text {cand }}$ size depending on the beam particle for two equivalents LET in the endothelium cell nucleus in order to study the influence of the radiation quality.

It can be observed that, for both LET, the number of clusters for a given size was found to be always higher in the case of a proton irradiation.

Table 3. $\mathrm{DSB}_{\text {cand }}$ per particle and micrometer as a function of the cluster size for two equivalents LET.

\begin{tabular}{ccccc}
\hline $\begin{array}{c}\text { Cluster } \\
\text { size }\end{array}$ & \multicolumn{2}{c}{$\sim 40 \mathrm{keV} / \mu \mathrm{m}$} & \multicolumn{2}{c}{$\sim 26 \mathrm{keV} / \mu \mathrm{m}$} \\
Proton & Alpha & Proton & Alpha \\
\hline 2 & 0.50 & 0.33 & 0.38 & 0.20 \\
3 & 0.46 & 0.23 & 0.30 & 0.13 \\
4 & 0.36 & 0.14 & 0.20 & 0.08 \\
5 & 0.25 & 0.07 & 0.12 & 0.04 \\
6 & 0.16 & 0.04 & 0.07 & 0.03 \\
7 & 0.10 & 0.02 & 0.04 & 0.01 \\
\hline
\end{tabular}

For both type of irradiations, $90 \%$ of clusters contain between 2 and 7 energy transfer points, the majority of them having small size. Indeed, the clusters containing 2 or 3 points represent more than $47 \%$ of the total number of clusters.

The main difference between the two radiation qualities is the evolution in the size frequency with LET. Indeed, a for proton irradiation, the proportion of "small clusters" (containing 2 or 3 points) increases significantly with decreasing LET and "bigger clusters" (containing 4 to 7 points) tend to decrease with decreasing LET. For an alpha irradiation, whatever the LET, the proportion of clusters for a given size is quite stable.

These results could suggest that a proton irradiation induces more complex damages difficult to be repaired compared to an alpha irradiation at equivalent LET.

\section{Conclusions}

The aim of the present work is to evaluate the influence of the DNA density and the radiation quality for proton and alpha irradiations regarding the number and the complexity of the possible direct damages to DNA. Simulations with proton and alpha beams within two new geometrical target models representing the DNA content of an endothelium and a fibroblast were performed using the Geant4-DNA Monte Carlo code processes. Nevertheless, as the DNA is distributed homogeneously on the nuclei, it is the DNA density which is studied and not the influence of the cell type.

The influence of the DNA density has been studied for protons and alpha irradiations. These results show that the quantity and the complexity of the evaluated potential damages in the endothelium cell nucleus are always higher than in the fibroblast cell nucleus. Regarding the influence of the radiation quality, for an equivalent LET, a proton irradiation induces more direct complex clustered damages than an alpha irradiation. These results suggest that the DNA density and the radiation quality influence the type and the quantity of damages. Moreover, proton irradiation seems to be more difficult to repair and could increase the radio-induced chromosomal aberrations or cell death compared to an alpha irradiation.

Nevertheless, this study concerns only the direct effects of ionizing radiation. As the computational tools for the simulation of the physico-chemical and the chemical stages needed for the evaluation of indirect effects are under development on Geant4 [14], the methodology presented here will be used to study the total effects on a future work.

Some radiobiological experiments on the two types of cell nuclei studied in this work are in progress in our Institute using proton and alpha beams. In these experiments an immunofluorescence technique is used in order to detect $\gamma-\mathrm{H} 2 \mathrm{AX}$ foci corresponding to radio-induced DSB. The experimental analysis of the cinematic of the foci appearance will help in verifying the conclusions obtained by Monte Carlo simulations presented in this work.

In parallel, a study concerning the influence of the euchromatin and heterochromatin regions is in progress. For this, the shape of chromatin fiber is modified (solenoid chromatin fiber conformation or nucleosomes placed randomly in the volume) in order to evaluate the influence of this parameter in the results of the number and complexity of direct clustered damages induced after protons and alpha irradiations.

\section{References}

[1] D.T. Goodhead, Initial events in the cellular effects of ionizing radiations: clustered damage in DNA, Int. J. Radiat. Biol. 65 (1994), pp. 7-17.

[2] D. Frankenberg, M. Frankenberg-Schwager, D. Blocher and R. Harbich, Evidence for DNA double strand breaks as the critical lesions yeast cells irradiated with sparsely or densely ionizing radiation under oxic or anoxic conditions, Radiat. Res. 88 (1981), pp. 524-532.

[3] S. Agostinelli, J. Allison, K. Amako et al., GEANT4-a simulation toolkit, Nucl. Instrum. Methods. Phys. Res. A. 506 (2003), pp. 250-303.

[4] J. Allison, K. Amako, J. Apostolakis et al., Geant4 developments and applications, IEEE Trans. Nucl. Sci. 53 (2006), pp. 270-278.

[5] C. Villagrasa, Z. Francis and S. Incerti, Physical models implemented in the GEANT4-DNA extension of the GEANT4 toolkit for calculating initial radiation damage at the molecular level, Radiat. Prot. Dosim. 143 (2011), pp. 214-218.

[6] S. Incerti, A. Ivanchenko, M. Karamitros, A. Mantero, P. Moretto, H. N. Tran, B. Mascialino, C. Champion, V. N. Ivanchenko, M. A. Bernal, Z. Francis, C. Villagrasa, G. Baldacchino, P. Guèye, R. Capra, P. Nieminen and C. Zacharatou, 
Comparison of GEANT4 very low energy cross section models with experimental data in water, Med. Phys. 37 (2010), pp. 4692-4708.

[7] Z. Francis, S. Incerti, M. Karamitros, H.N. Tran and C. Villagrasa, Stopping power and ranges of electrons, protons and alpha particles in liquid water using the GEANT4-DNA package, Nucl. Instrum. Methods Phys. Res. B. 269 (2011), pp.2307-2311.

[8] Z. Francis, S. Incerti, R. Capra, B. Mascialino, G. Montarou, V. Stepan and C. Villagrasa, Molecular track scale structure simulations in liquid water using the GEANT4-DNA Monte Carlo code processes, Appl. Radiat. Isot. 69 (2011), pp. 220-226.

[9] W. Friedland, P. Jacob, H.G. Paretzke and T. Stork, Monte Carlo simulation of the production of short DNA fragments by low linear energy transfer radiation using higher order DNA models, Radiat. Res. 150 (1998), pp. 170-182.

[10] W. Friedland, M. Dingfelder, P. Kundrat and P. Jacob, Track structures, DNA targets and radiation effects in the biophysical Monte Carlo simulation code PARTRAC, Muta. Res. 711 (2011), pp. 28-40.

[11] M. Dos Santos, C. Villagrasa, I. Clairand and S. Incerti, Influence of the DNA density in the number of clustered damages created by protons of different energies, accepted in Nucl. Instrum. Methods Phys. Res. B.

[12] Z. Francis, C. Villagrasa and I. Clairand, Simulation of DNA damage algorithm after proton irradiation using and adapted DBSCAN algorithm, Comput. Methods. Programs Biomed.101 (2011), pp. 265-670.

[13] Z. Francis, S. Incerti, V. Ivanchenko, C. Champion, M. Karamitros, M. A. Bernal and Z. El Bitar, Monte Carlo simulation of energy deposit clustering for ions of the same LET in liquid water, Phys. Med. Biol. 57 (2012), pp. 209-224.

[14] M. Karamitros, A. Mantero, S. Incerti et al., Modelling radiation chemistry in the GEANT4 toolkit, Nucl. Sci. Tech. 2 (2011) 503-508. 